\title{
Abandono precoce da consulta de cessação tabágica da USF do Parque
}

Sofia Belo, ${ }^{1}$ Luís Rebelo ${ }^{2-3}$

\section{RESUMO}

Objetivos: Caracterizar os utentes da consulta de cessação tabágica da USF do Parque (ACeS Lisboa Norte) e taxa de abandono precoce. Identificar os fatores associados ao abandono precoce.

Tipo de estudo: Estudo observacional retrospetivo.

Local: USF do Parque - ACeS Lisboa Norte.

População: Foram estudados todos os fumadores que iniciaram o programa de consultas de cessação tabágica da USF do Parque no ano 2013 (total de 83 fumadores).

Métodos: Os dados foram obtidos através da análise documental das fichas clinicas da consulta. Foi feita uma análise estatística básica para caracterizar a população e, posteriormente, de acordo com o comportamento dos fumadores após a primeira consulta de cessação tabágica, foi possível definir dois subgrupos: os que "abandonaram o programa após a primeira consulta (abandono precoce)" e os que se "mantiveram no programa". Estes dois subgrupos foram submetidos a uma análise comparativa através da aplicação dos testes de Qui-quadrado e Exato de Fisher. Foi considerado um intervalo de confiança de 95\%.

Resultados: Amostra de 83 fumadores, com discreto (50,6\%) predomínio do sexo masculino e com média de idades de 47,8 anos, a maioria licenciados e atualmente empregados. O início do consumo de tabaco data do período da adolescência (média 16,2 anos), presentemente rondando os vinte cigarros por dia. Em 59,0\% dos fumadores apurou-se um nível moderado de dependência de nicotina. Também relativamente à motivação, o nível moderado foi predominante $(58,7 \%)$. Aproximadamente um terço desistiu do programa após a primeira consulta (abandono precoce). Verificou-se uma associação estatisticamente significativa entre o abandono precoce e as variáveis psicopatologia $(p=0,02)$ e problemas familiares $(p=0,03)$.

Conclusões: Os programas de apoio intensivo à cessação tabágica exigem um contacto contínuo, regular e muitas vezes prolongado entre médico-fumador; no entanto, as desistências são comuns, particularmente após a primeira consulta. Na literatura, quer a psicopatologia quer os problemas familiares, sabiam-se estar associados ao sucesso da intervenção; agora percebe-se que também têm um impacto a curto prazo estando implicados no abandono precoce. Este resultado salienta a importância do primeiro contacto médicofumador e reflete a necessidade de melhorar a avaliação global realizada e de aperfeiçoar as estratégias de apoio e seguimento no intuito de garantir a assiduidade dos utentes.

Palavras-chave: Tabaco; Consulta; Medicina geral e familiar; Abandono do hábito de fumar

\section{INTRODUÇÃO}

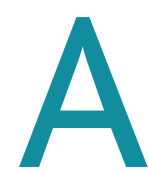
epidemia do tabagismo é hoje considerada uma das maiores ameaças à saúde pública, na medida em que as suas consequências se traduzem em repercussões em toda a população (fumadora e não fumadora). ${ }^{1}$

1. Médica Interna de formação específica do $1^{\circ}$ ano de Medicina Geral e Familiar. USF Santiago de Palmela, ACeS Arrábida.

2. Médico responsável pela consulta de cessação tabágica. USF do Parque, ACeS Lisboa Norte.

3. Professor auxiliar. Faculdade de Medicina e Instituto de Medicina Preventiva, Universidade de Lisboa.
De acordo com estimativas da OMS, o tabaco é responsável por cerca de seis milhões de mortes por ano, 600.000 das quais se devem à exposição ao fumo ambiental. ${ }^{2}$ Para além deste pesado impacto na mortalidade, fumar surge também como a primeira causa evitável de doença e incapacidade no mundo, sendo ainda responsável por retirar anos de vida saudável aos consumidores. ${ }^{1,3-5}$

Face a esta iminente ameaça foram implementadas diversas estratégias a nível global, entre as quais a Convenção-Quadro para o Controlo do Tabaco em 2003 e, a nível nacional, em 2012, a Prevenção e Controlo do Tabagismo integra um dos programas de saúde prioritários do Plano 
Nacional de Saúde 2012-2016, ${ }^{4}$ tendo como principais objetivos:

1. Prevenção do consumo;

2. Promoção da cessação;

3. Proteção da exposição ambiental;

4. Criação de um clima social, cultural e económico favorável à adoção de estilos de vida saudável.

Dados do Inquérito Nacional de Saúde realizado em 2014 traduzem já uma evolução positiva onde, apesar de a população fumadora se manter sobreponível à encontrada em 2005/06, se nota um aumento significativo na percentagem de não fumadores: de 16 para $21,9 \%{ }^{6}$

De todas as medidas implicadas no controlo do tabagismo, a cessação tabágica é o gold standard,$^{1,4,7}$ particularmente as intervenções de apoio intensivo que englobam um programa estruturado de consultas, desenvolvidas por profissionais especializados que garantem um contacto interpessoal regular e disponibilizando estratégias de tratamento farmacológico e de terapia cognitivo-comportamental personalizadas ao contexto global do fumador avaliado na primeira consulta. ${ }^{1,5}$

No entanto, e apesar do investimento nestes programas, existe uma elevada taxa de abandono, particularmente marcada após a primeira consulta, ${ }^{8-9}$ que se sabe ser decisiva para o sucesso da intervenção. ${ }^{1,7}$

Neste âmbito, e no intuito de trazer uma nova vertente de avaliação na área da cessação tabágica, o presente estudo foi delineado com o objetivo de caracterizar a taxa de abandono precoce (abandono imediatamente após a primeira consulta) e compreender os fatores associados à mesma.

\section{MÉTODOS}

A consulta de cessação tabágica da USF do Parque (ACeS Lisboa Norte) iniciou-se em 2002 e desde essa data que se mantém em funcionamento, atualmente em horário semanal, às terças-feiras (8-11 horas), sendo da responsabilidade de uma equipa constituída por um médico e uma enfermeira com o apoio esporádico de uma psicóloga.

No intuito de caracterizar a taxa de abandono imediatamente após a primeira consulta de cessação tabágica (abandono precoce) realizou-se um estudo de coorte retrospetivo, tendo por base a análise documental das fichas clínicas de todos os inscritos na consulta de cessação tabágica da USF do Parque (ACeS Lisboa Norte), no ano de 2013. Obteve-se uma amostra de 83 fumadores.

As fichas clínicas desta consulta estão estruturadas de acordo com o Programa tipo de Cessação Tabágica ${ }^{1}$ e, de acordo com os dados de preenchimento disponíveis, foi possível avaliar as seguintes variáveis: sexo, idade, escola- ridade, situação profissional e familiar, apoios de recurso, presença de psicopatologia, história tabágica (idade de início, número de cigarros por dia, nível de dependência da nicotina, existência ou não de tentativas prévias), motivação para deixar de fumar e plano de atuação implementado (farmacológica e não farmacológica).

Algumas variáveis constantes do formulário foram adaptadas, nomeadamente a variável «Apoios de recurso» foi definida como «todo o apoio social disponível para o fumador, fora do contexto das consultas», conceito adaptado do trabalho realizado por Soares e Carneiro, ${ }^{7}$ sendo criadas as classes «Sim»/ «Não» e discriminadas as subcategorias: «Parceiro(a)», «Filho(s)», «Parceiro e filho(s)», «Amigo(s)/Colegas», «Pais» $\mathrm{e}$ «Outros».

No presente artigo considerou-se «Psicopatologia» a presença de: anorexia nervosa/bulimia, depressão/doença bipolar ou outra (se existisse referência aos diagnósticos de ansiedade, esquizofrenia/psicose, perturbações da personalidade). Foram excluídos os casos de outras dependências (alcoolismo, toxicodependência ou outras) e também casos em que se apurou prescrição de psicofármacos na ausência de diagnóstico formal de psicopatologia (e.g., no contexto de patologia de outro foro: neurológico, perturbações do sono) pelo facto de poderem atuar como fatores de confundimento.

Os dados recolhidos foram registados e analisados recorrendo ao programa Microsoft Excel. Primeiramente foi feita uma análise estatística simples, com cálculos de frequências absolutas e relativas e medidas de tendência central e dispersão. Posteriormente, e tendo definido abandono precoce como aquele que ocorria após a primeira consulta, foi possível definir dois subgrupos de fumadores: «os que abandonaram precocemente» $\mathrm{e}$ «os que continuaram no programa».

No intuito de verificar se existia uma associação entre a possibilidade de abandonar precocemente o programa de consultas e alguma das variáveis em estudo foram aplicados testes não paramétricos, na maioria dos casos o teste de Qui-Quadrado. Todavia, quando não eram cumpridos os critérios necessários para aplicar o teste de QuiQuadrado, nomeadamente para as variáveis «Apoios de recurso» e «Proposta terapêutica», aplicou-se o teste Exato de Fisher. Em todas as análises efetuadas foi considerado um intervalo de confiança de $95 \%$.

\section{RESULTADOS}

Caracterização dos fumadores e ambiente envolvente

No Quadro I estão representadas algumas das caracterís- 
ticas dos fumadores. Foram estudados 83 fumadores, 41 $(49,4 \%)$ eram do sexo feminino e $42(50,6 \%)$ do sexo masculino. A média de idades foi de 47,8 anos, com um mínimo de 15 e um máximo de 85 anos. Distribuídos por grupos etários, o grupo dos 46-55 anos foi o mais frequente com $28,9 \%$.

A maioria dos fumadores tinha licenciatura (40,9\%), integrava a população ativa $(61,4 \%)$ e negava a existência de problemas profissionais recentes $(66,3 \%)$. É de salientar a baixa taxa de desempregados (16,9\%).

Tendo em conta os critérios utilizados neste trabalho para definir a variável «Psicopatologia» na presente população apurou-se a presença de psicopatologia em 25,3\% dos fumadores, salientando-se a depressão como o diagnóstico mais prevalente $(71,4 \%)$.

Em relação à situação familiar, a maioria negava a existência de problemas recentes $(74,7 \%)$. Apenas $42,2 \%$ mencionou ter apoios de recurso disponíveis, salientando-se a elevada percentagem de omissos em relação ao preenchimento deste parâmetro.

\section{História tabágica}

No Quadro II estão representados os dados relativos aos hábitos tabágicos dos fumadores estudados.

A idade média de consumo do primeiro cigarro, calculada com base nos dados individuais, foi de 16,2 anos, registando-se um mínimo de nove e um máximo de 37 anos. Este valor está de acordo com a distribuição dos fumadores por faixa etária, em que o grupo entre os 15 e os 19 anos foi o mais frequente $(56,6 \%)$.

A maioria dos fumadores mantinha o consumo em média há 31,1 anos, fumando atualmente, também em média, 20 cigarros por dia.

Avaliando o nível de dependência de nicotina com o Teste de Fagerström, e posteriormente agregando os resultados nos três subgrupos de dependência, obteve-se que $59,0 \%$ dos fumadores se situava no nível moderado e apenas $14,5 \%$ no elevado.

Mais de metade (54,2\%) dos fumadores já tinha efetuado previamente tentativas para abandonar o consumo de tabaco.

\section{Motivação}

Para avaliar o nível de motivação para deixar de fumar recorre-se ao Teste de Richmond e de acordo com a pontuação obtida, numa escala de 0-10, agrupam-se em três subgrupos: motivação baixa, moderada e alta. Na população estudada, a pontuação média foi de 7,2 pontos, sendo que a maioria $(68,7 \%)$ dos fumadores integravam o sub-

\begin{tabular}{|c|c|c|}
\hline \multicolumn{3}{|c|}{$\begin{array}{l}\text { QUADRO I. Caracterização dos fumadores e ambiente } \\
\text { envolvente }\end{array}$} \\
\hline & $\mathrm{n}$ & $\%$ \\
\hline \multicolumn{3}{|c|}{ Sexo } \\
\hline Masculino & 42 & 50,6 \\
\hline Feminino & 41 & 49,4 \\
\hline \multicolumn{3}{|c|}{ Faixa etária } \\
\hline$\leq 15$ Anos & 1 & 1,2 \\
\hline $16-25$ Anos & 6 & 7,2 \\
\hline $26-35$ Anos & 7 & 8,4 \\
\hline $36-45$ Anos & 20 & 24,1 \\
\hline $46-55$ Anos & 24 & 28,9 \\
\hline $56-65$ Anos & 19 & 22,9 \\
\hline$\geq 65$ Anos & 6 & 7,2 \\
\hline \multicolumn{3}{|c|}{ Escolaridade } \\
\hline Analfabeto & 0 & 0 \\
\hline Primária & 7 & 8,4 \\
\hline Secundária & 26 & 31,3 \\
\hline Média & 7 & 8,4 \\
\hline Licenciatura & 34 & 40,9 \\
\hline Omissos & 9 & 10,8 \\
\hline \multicolumn{3}{|c|}{ Situação profissional } \\
\hline Ativa & 51 & 61,4 \\
\hline Em formação & 3 & 3,6 \\
\hline Reforma & 13 & 15,7 \\
\hline Desemprego & 14 & 16,9 \\
\hline Omissos & 2 & 2,4 \\
\hline \multicolumn{3}{|c|}{ Problemas profissionais recentes? } \\
\hline Não & 55 & 66,3 \\
\hline Sim & 23 & 27,7 \\
\hline Omissos & 5 & 6,0 \\
\hline \multicolumn{3}{|c|}{ Psicopatologia } \\
\hline Não & 59 & 71,1 \\
\hline Sim: & 21 & 25,3 \\
\hline Depressão & 15 & 71,4 \\
\hline Doença bipolar & 0 & 0 \\
\hline Anorexia/Bulimia & 1 & 4,8 \\
\hline Outra & 5 & 23,8 \\
\hline Omissos & 3 & 3,6 \\
\hline \multicolumn{3}{|c|}{ Problemas familiares recentes? } \\
\hline Sim & 14 & 16,9 \\
\hline Não & 62 & 74,7 \\
\hline Omissos & 7 & 8,4 \\
\hline \multicolumn{3}{|c|}{ Apoios de recurso } \\
\hline Não & 10 & 12,0 \\
\hline Sim: & 35 & 42,2 \\
\hline Parceiro(a) & 10 & 28,6 \\
\hline Filho(s) & 11 & 31,4 \\
\hline Parceiro(a) e filho(s) & 10 & 28,6 \\
\hline Amigo(s)/colega(s) & 3 & 8,6 \\
\hline Pais & 1 & 2,9 \\
\hline Omissos & 38 & 45,8 \\
\hline
\end{tabular}


grupo «Motivação moderada» (Quadro III). Infelizmente, em nenhum se registou a pontuação máxima.

\section{Estratégia proposta}

Em praticamente 93\% dos fumadores optou-se pela estratégia combinada de terapia cognitivo-comportamental (TCC) e tratamento farmacológico. Em cinco dos fumadores estudados recorreu-se a TCC exclusiva (Quadro IV). Salienta-se o facto de não se ter utilizado a opção farmacológica como método isolado como preconizado na literatura.

\section{Abandono precoce}

O abandono está patente ao longo de todo o programa e cerca de um terço dos fumadores que iniciaram o programa em 2013 abandonaram imediatamente após a primeira consulta, ou seja, precocemente (Diagrama 1). No intuito de perceber quais os fatores relacionados com o abandono precoce das consultas de cessação tabágica na população em estudo criou-se o Quadro V, que compara as características dos dois grupos de utentes. Apenas se apurou uma associação estatisticamente significativa entre o abandono precoce e as variáveis «Psicopatologia»e «Problemas recentes», com valores $p$ de 0,02 e 0,03 , respetivamente.

\section{DISCUSSÃO}

Em 2013, o total de inscritos na consulta de cessação tabágica da USF do Parque foi de 83 indivíduos, distribuídos de forma praticamente equitativa de acordo com o género. A média de idades da população (47,8 anos) foi ligeiramente superior à encontrada noutros estudos, ${ }^{8,10-11}$ provavelmente pelo facto de os indivíduos de idade mais avançada serem os que mais recorrem aos cuidados de saúde primários, ${ }^{13}$ tendo por isso uma maior probabilidade de ser aconselhados a deixar de fumar e informados acerca da existência das consultas.

O facto de se ter optado, embora intencionalmente, por incidir o estudo apenas sobre os inscritos na consulta de cessação tabágica da USF do Parque leva a que a população estudada espelhe a população de uma área geográfica circunscrita, neste caso, a área englobada pelo ACeS Lisboa Norte com predomínio dos inscritos no Centro de Saúde de Alvalade. Por este motivo, não é de estranhar a elevada taxa de licenciados e população ativa e a baixa percentagem de desempregados encontrada; aliás, estes dados são concordantes com os obtidos por Rebelo ${ }^{10}$ no estudo realizado em 2008, incidindo na mesma população. Não obstante, é necessário considerar que esta situação gera um viés que poderá ter tido impacto nos resultados objetiva-

\begin{tabular}{|c|c|c|}
\hline \multicolumn{3}{|c|}{$\begin{array}{l}\text { QUADRO II. Caracterização da história e hábitos } \\
\text { tabágicos }\end{array}$} \\
\hline & $n$ & $\%$ \\
\hline \multicolumn{3}{|c|}{ Idade do primeiro cigarro } \\
\hline$<10$ Anos & 1 & 1,2 \\
\hline $10-14$ Anos & 23 & 27,7 \\
\hline $15-19$ Anos & 47 & 56,6 \\
\hline $20-24$ Anos & 8 & 9,6 \\
\hline $25-29$ Anos & 0 & 0 \\
\hline$\geq 30$ Anos & 1 & 1,2 \\
\hline Omissos & 3 & 3,6 \\
\hline \multicolumn{3}{|c|}{ Número de anos de consumo } \\
\hline$<10$ Anos & 7 & 8,4 \\
\hline $10-19$ Anos & 7 & 8,4 \\
\hline $20-29$ Anos & 22 & 26,5 \\
\hline$\geq 30$ Anos & 45 & 54,2 \\
\hline Omissos & 2 & 2,4 \\
\hline \multicolumn{3}{|c|}{ Número de cigarros por dia } \\
\hline$<10$ Cigarros/dia & 6 & 7,2 \\
\hline 10 - 19 Cigarros/dia & 30 & 36,1 \\
\hline 20 - 29 Cigarros/dia & 25 & 30,1 \\
\hline 30 - 39 Cigarros/dia & 10 & 12,1 \\
\hline$\geq 40$ Cigarros/dia & 8 & 9,6 \\
\hline Omissos & 4 & 4,8 \\
\hline \multicolumn{3}{|c|}{ Nível de dependência de nicotina - Teste de Fagerstrom } \\
\hline Baixa $(<4)$ & 20 & 24,1 \\
\hline Moderada $(4-7)$ & 49 & 59,0 \\
\hline Elevada $(>7)$ & 12 & 14,5 \\
\hline Omissos & 2 & 2,4 \\
\hline \multicolumn{3}{|c|}{ Tentativas anteriores para deixar de fumar } \\
\hline Sim & 45 & 54,2 \\
\hline Não & 36 & 43,4 \\
\hline Omissos & 2 & 2,4 \\
\hline
\end{tabular}

\begin{tabular}{|c|c|c|}
\hline \multicolumn{3}{|c|}{$\begin{array}{l}\text { QUADRO III. Motivação para deixar de fumar (Teste de } \\
\text { Richmond) }\end{array}$} \\
\hline & $n$ & $\%$ \\
\hline Baixa $(0-6)$ & 22 & 26,5 \\
\hline Moderada (7 - 9) & 57 & 68,7 \\
\hline Alta (10) & 0 & 0 \\
\hline Omissos & 4 & 4,8 \\
\hline
\end{tabular}




QUADRO IV. Estratégia proposta
\begin{tabular}{l|r|c} 
\\
\hline Cognitivo-comportamental & $n$ & $\%$ \\
\hline Farmacológica & 5 & 6 \\
\hline Combinada* & 0 & 0 \\
\hline Outra & 77 & 92,8 \\
\hline
\end{tabular}

*Estratégia combinada de terapia cognitivo-comportamental e tratamento farmacológico.

dos, limitando, deste modo, a generalização das conclusões obtidas. Nesse âmbito, seria bastante pertinente desenvolver futuras investigações incidindo numa maior e mais heterogénea população no intuito de minimizar o impacto deste enviesamento nas hipóteses levantadas e permitir conclusões com maior implicação científica.

Também é importante salientar que a maioria dos fumadores negava problemas profissionais e familiares e admitia ter apoios de recurso disponíveis, aspetos estes que traduzem um ambiente adequado e favorável para o processo de mudança de estilo de vida que a cessação tabágica implica.

A literatura reconhece que a presença de psicopatologia é fator de insucesso para o abandono do consumo do tabaco. ${ }^{1,14}$ Por essa razão, a avaliação de comorbilidades, particularmente patologia do foro mental, é um parâmetro essencial na avaliação global do fumador levada a cabo na primeira consulta de cessação tabágica. No presente estudo apurou-se a existência de psicopatologia em cerca de $25 \%$ dos fumadores, resultado semelhante ao observado por Fonseca e colaboradores $(28,9 \%)^{13}$ e por Rebelo $(31 \%),{ }^{10} \mathrm{em}-$ bora inferior ao registado por Aguiar e colaboradores $(45,6 \%) .{ }^{8}$ No entanto, há que ressalvar que não existem cri- térios específicos que definam o conceito de psicopatologia e que, apesar de existirem escalas que podem ser utilizadas para o efeito, estas nem sempre são aplicadas e por este motivo podem existir considerações diferentes entre autores que justifiquem esta discrepância de achados. Dentro da patologia psiquiátrica, a depressão foi a mais prevalente $(71,4 \%)$ como encontrado por Aguiar e colaboradores. ${ }^{8}$

Relativamente aos hábitos tabágicos, os dados corroboram com os de outros autores, ${ }^{8,10,13}$ apurando-se um início do consumo na fase da adolescência e uma manutenção desde então. Estes resultados alertam para a necessidade, no intuito da prevenção primária, de intervir junto das camadas mais jovens para eliminar esta tendência. ${ }^{1,4,8,11}$

No que diz respeito ao grau de dependência de nicotina, a maioria da população estudada situava-se no nível moderado e apenas 14,5\% no elevado. Quanto à motivação, os resultados são semelhantes, sendo o nível moderado o mais frequente, resultados estes análogos a outros estudos. ${ }^{10,13}$

A existência de tentativas prévias para deixar de fumar sabe-se ser um fator preditivo para o sucesso da cessação tabágica. ${ }^{1,5,7}$ No entanto, no presente estudo, apesar de mais de metade dos fumadores terem tido tentativas prévias de abandono do consumo do tabaco, este contexto não teve impacto na taxa de abandono precoce, como talvez seria de esperar. Apesar de podermos referir que, enquanto variável isolada, não se verificou uma associação com significância estatística entre as tentativas prévias e o abandono precoce das consultas, como não foi realizada uma análise multivariada não se pode afirmar, nem tão pouco excluir, que esta variável não tenha importância quando atuando em conjunto com outra(s) em simultâneo. Esta limitação é inerente a todas as variáveis estudadas neste trabalho, uma vez que foram analisadas de forma isolada e individual, excluindo-se a hipótese de se poderem correlacionar entre si e, deste modo,

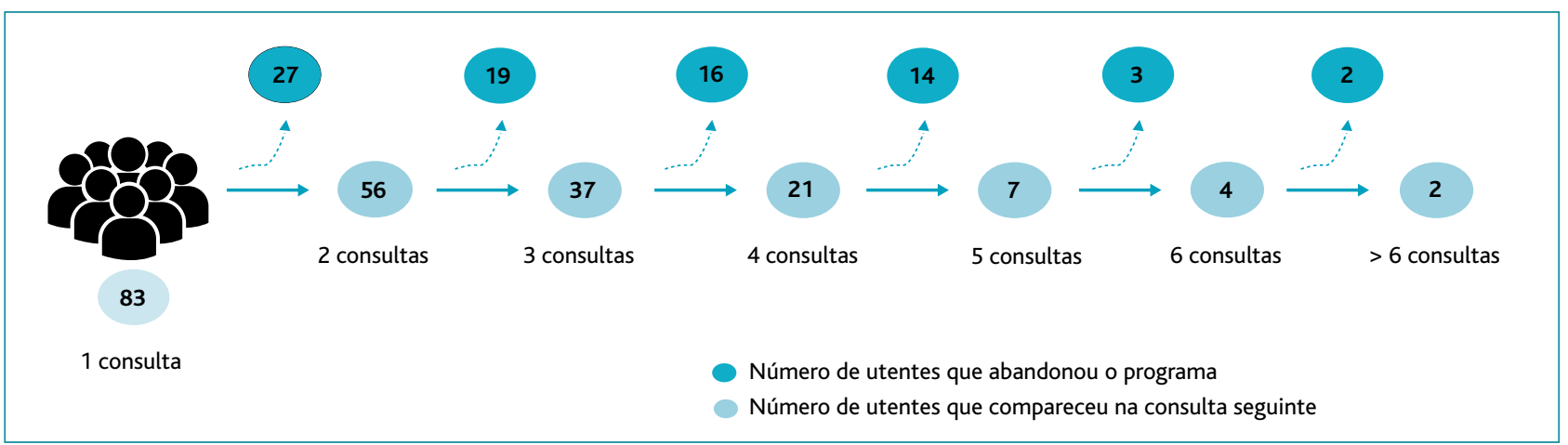

Diagrama 1. Evolução do número de doentes ao longo das consultas de CT. 


\begin{tabular}{|c|c|c|c|c|c|c|}
\hline \multicolumn{7}{|c|}{$\begin{array}{l}\text { QUADRO V. Comparação das características entre o grupo de fumadores } \\
\text { que abandonou precocemente e o grupo que continuou no programa }\end{array}$} \\
\hline & & \multicolumn{2}{|c|}{$\begin{array}{l}\text { Abandonaram } \\
\text { precocemente }\end{array}$} & \multicolumn{2}{|c|}{$\begin{array}{l}\text { Continuaram } \\
\text { em programa }\end{array}$} & \multirow[t]{2}{*}{ Valor $p$} \\
\hline & & $n$ & $\%$ & $n$ & $\%$ & \\
\hline \multirow[t]{2}{*}{ Sexo } & Feminino & 15 & 55,6 & 26 & 46,4 & $0,44^{*}$ \\
\hline & Masculino & 12 & 44,4 & 30 & 53,6 & \\
\hline \multirow[t]{2}{*}{ Escolaridade } & $<$ Ensino superior* & 15 & 62,5 & 25 & 50 & 0,31 \\
\hline & Ensino superior & 9 & 37,5 & 25 & 50 & \\
\hline \multirow{2}{*}{$\begin{array}{l}\text { Situação } \\
\text { profissional }\end{array}$} & Ativo & 14 & 56 & 36 & 64,3 & 0,48 \\
\hline & Não ativo & 11 & 44 & 20 & 35,7 & \\
\hline \multirow{2}{*}{$\begin{array}{l}\text { Problemas no } \\
\text { trabalho }\end{array}$} & Sim & 5 & 19,2 & 18 & 34,6 & 0,16 \\
\hline & Não & 21 & 80,8 & 34 & 65,4 & \\
\hline \multirow{2}{*}{$\begin{array}{l}\text { Apoios de } \\
\text { recurso** }\end{array}$} & Sim & 8 & 61,5 & 27 & 84,4 & 0,12 \\
\hline & Não & 5 & 38,5 & 5 & 15,6 & \\
\hline \multirow{2}{*}{$\begin{array}{l}\text { Problemas } \\
\text { familiares }\end{array}$} & Sim & 8 & 32 & 6 & 11,8 & 0,03 \\
\hline & Não & 17 & 68 & 45 & 88,2 & \\
\hline \multirow[t]{2}{*}{ Psicopatologia } & Sim & 11 & 42,3 & 10 & 18,5 & 0,02 \\
\hline & Não & 15 & 57,7 & 44 & 81,5 & \\
\hline \multirow{2}{*}{$\begin{array}{l}\text { Tentativas } \\
\text { prévias }\end{array}$} & Sim & 17 & 63 & 28 & 51,9 & 0,34 \\
\hline & Não & 10 & 37 & 26 & 48,1 & \\
\hline \multirow{2}{*}{$\begin{array}{l}\text { Nível de } \\
\text { dependência }\end{array}$} & Baixa & 8 & 29,6 & 12 & 22,2 & 0,47 \\
\hline & Mod/alta & 19 & 70,4 & 42 & 77,8 & \\
\hline \multirow{2}{*}{$\begin{array}{l}\text { Nível de } \\
\text { motivação }\end{array}$} & Baixa & 8 & 33,3 & 13 & 24,1 & 0,39 \\
\hline & Mod/alta & 16 & 66,7 & 41 & 75,9 & \\
\hline \multirow[t]{2}{*}{$\begin{array}{l}\text { Proposta } \\
\text { terapêutica** }\end{array}$} & $\begin{array}{l}\text { Com recuso a } \\
\text { fármacos }\end{array}$ & 24 & 88,9 & 53 & 96,4 & 0,33 \\
\hline & $\begin{array}{l}\text { Sem recurso a } \\
\text { fármacos }\end{array}$ & 3 & 11,1 & 2 & 3,6 & \\
\hline
\end{tabular}

*Nesta variável estão incluídas as categorias: analfabeto, escolaridade primária, secundária e média. **Nestas variáveis foi aplicado o Teste exato de Fisher.

influenciar os resultados. De facto, esta é uma importante barreira deste estudo; apesar disso, dá a oportunidade de desenvolver outros projetos a fim de colmatar esta lacuna.

Em relação às medidas propostas verifica-se que a abordagem preferencial foi a estratégia combinada de terapia cognitivo-comportamental e tratamento farmacológico, como é aliás fortemente recomendado na literatura devido à sua maior eficácia ${ }^{1,5,7,12} \mathrm{e}$ uma vez que atua nas principais vertentes da dependência tabágica: comportamental, psicológica e física.

Embora a maioria da investigação clínica na área da cessação tabágica incida na avaliação dos resultados das in- tervenções (sucesso ou insucesso na tentativa de deixar de fumar), raramente existe uma preocupação em compreender o elevado número de desistências registadas, ainda que esta problemática seja crucial tendo em conta a estreita relação que existe entre a intensidade do aconselhamento e o êxito dos programas. $^{7}$ Apesar das limitações existentes, o presente trabalho tencionou ir mais além e debruçar-se sobre o abandono das consultas em maior profundidade.

Com a análise longitudinal efetuada foi possível demonstrar que $o$ abandono foi transversal ao longo do programa de consultas; no entanto, e corroborando com outros trabalhos nacionais, ${ }^{8-9}$ foi particularmente marcado após a primeira consulta onde se registou quase um terço de desistências. Este achado questionou eventuais fatores subjacentes passíveis de identificar e, a posteriori modificar ou mesmo evitar, no intuito de garantir a assiduidade do doente às consultas programadas e a manutenção da vontade e motivação para atingir a meta a que se propôs na primeira avaliação: deixar de fumar. De entre as variáveis estudadas nos dois grupos de fumadores («Abandonaram precocemente» $\mathrm{e}$ "Continuaram em programa») apenas se apurou uma associação com significância estatística entre abandono precoce e psicopatologia e problemas familiares, com valores $p$ de 0,02 e 0,03 , respetivamente. É ainda importante referir que não foi realizada nenhuma análise para averiguar se as duas variáveis associadas ao abandono precoce estavam associadas entre si (i.e., se existia alguma relação de dependência entre psicopatologia e problemas familiares). Contudo, esta lacuna dá a oportunidade de se desenvolverem outros trabalhos no intuito de verificar esta associação e, assim, enriquecer estes resultados.

Não obstante, o facto de se ter incidido o estudo num período de tempo curto (um ano) e numa área geográfica circunscrita (inscritos na consulta de cessação tabágica da USF do Parque) resultou numa reduzida e homogénea amostra, o que impossibilita a generalização dos resultados, pelo que seria pertinente alargar não só o período de estudo como di- 
versificar a população analisada, por exemplo, estudando também consultas de cessação tabágica de outros centros de saúde ou até a nível hospitalar. Ainda assim, o presente estudo expõe a necessidade de melhorar a caracterização de alguns parâmetros da avaliação global do fumador realizada na primeira consulta e de aperfeiçoar o programa de apoio e o acompanhamento prestados aos fumadores.

Na presença de patologia do foro psíquico, não só a prevalência de tabagismo é maior como existe uma menor taxa de sucesso em abandonar o consumo..$^{1,3,8,10,13-14}$ Em parte, esta correlação negativa pode ser explicada pelo facto destes doentes recorrerem ao tabaco para alívio de sintomas, situação particularmente frequente no caso da depressão em que «fumar» é muitas vezes fonte de prazer. ${ }^{3,14}$ O tabaco, por sua vez, tratando-se de uma dependência, na sua vertente biológica modifica os mecanismos de neurotransmissores, tendo presumivelmente impacto na própria patologia mental. .,14 $^{3}$

São vários os estudos que apontam para o facto do tabagismo e da psicopatologia se puderem influenciar mutuamente quando coexistem no mesmo indivíduo,,$^{1,3,8,10,13-14}$ ou seja, o tabagismo pode atuar no quadro psicopatológico, o qual, por sua vez, pode afetar a dependência tabágica. ${ }^{14}$ Devido a esta interação, a cessação tabágica nesta população conota-se quer por síndromas de abstinência mais marcadas e difíceis de tolerar, quer pela exacerbação da psicopatologia de base, ${ }^{1}$ exigindo por isso um acompanhamento e um aconselhamento adaptados às características destes doentes. Todas estas especificidades explicam a elevada probabilidade de recaída e consequente insucesso no abandono do tabaco registada neste subgrupo de fumadores noutras investigações. ${ }^{1,3,5,14}$ Apoiam igualmente os resultados objetivados no presente estudo na medida em ajudam a justificar o maior abandono precoce dos programas de apoio intensivo nestes indivíduos e, ainda, que não seja possível com a presente investigação saber especificamente quais as razões para tal; admite-se que possa presumivelmente estar relacionado com uma de três situações, ou várias em simultâneo: o quadro de abstinência sentido (particularmente na fase inicial de cessação tabágica), a descompensação da psicopatologia de base ou o timing desadequado para iniciar uma tentativa.

Estes resultados salientam a importância de procurar a presença de psicopatologia em todos os fumadores que se inscrevem em programas intensivos de apoio à cessação, especialmente na fase inicial onde é necessário que o médico responsável pela consulta seja capaz de avaliar o risco-benefício da intervenção para aquele indivíduo, naquele momento e naquelas condições. Nestes casos, a re- gra, antes de iniciar o programa de cessação tabágica, é garantir que o doente está estabilizado do ponto de vista mental e só depois considerar uma tentativa, já que deste modo se aumenta o êxito da última. ${ }^{14}$

A associação entre abandono precoce e psicopatologia reforça também o já defendido na literatura, em que o acompanhamento por uma equipa multidisciplinar é de extrema importância, principalmente em determinados grupos de fumadores como os portadores de psicopatologia. ${ }^{1,5,14}$ Entre outras particularidades, nestes fumadores as estratégias cognitivo-comportamentais merecem maior ênfase, ${ }^{14}$ na medida em que fornecem as ferramentas necessárias para lidar com situações em que a vontade de fumar é maior, prevenindo as recaídas que se conhecem superiores nestes indivíduos. ${ }^{1}$

Um ambiente familiar favorável de entreajuda e compreensão é um componente imprescindível no tratamento de qualquer doença e no caso do tabagismo, por se tratar de uma dependência, o apoio familiar torna-se ainda mais preponderante. ${ }^{1,15-17}$ Assim sendo, é lógico pressupor que a presença de instabilidade ou problemas familiares possa surgir como fonte de stress para o fumador a tentar deixar de fumar, aliciando-o a recair e a abandonar precocemente a tentativa. Na mesma linha de pensamento, uma meta-análise internacional ${ }^{16}$ mostrou que os fumadores casados tinham mais sucesso na tentativa de deixar de fumar que os divorciados, viúvos ou solteiros, sugerindo que o apoio e a disponibilidade do cônjuge aumentam o êxito dos programas de apoio intensivo. Todavia, no presente estudo não foi possível tecer considerações em relação a este aspeto já que nem o estado civil nem o agregado familiar constam da ficha de avaliação global do fumador utilizada na consulta de cessação tabágica da USF do Parque.

Alguns estudos ${ }^{3,15}$ destacam ainda que, além do suporte social ser fundamental na prevenção de recaídas, é inclusive imprescindível antes de iniciar o processo de cessação, nomeadamente na ação de incutir o desejo de mudança. Este fenómeno é particularmente importante naqueles fumadores que põem em causa a sua própria intenção e motivação; nestes, ter o voto de confiança das pessoas que lhes são significativas aumenta não só a probabilidade de tentar como o êxito em atingir o objetivo final. ${ }^{15}$

Tal como se encontrou associação entre abandono precoce e problemas familiares seria de esperar encontrar também para os apoios de recurso, já que os conceitos muitas das vezes se sobrepõem. Se se entende como apoio de recurso «todo o apoio social disponível para o fumador, fora do contexto das consultas», ${ }^{7}$ alterações da dinâmica familiar são um 
critério incluído no próprio conceito. Ainda assim, não se apurou uma associação estatisticamente significativa entre esta variável e o abandono precoce das consultas. Uma vez mais, é necessário lembrar que a pequena amostra pode justificar estas discrepâncias; neste caso ainda mais notória pelo facto de, em 38 fumadores, não haver qualquer registo no parâmetro «Apoios de recurso» na ficha de avaliação.

Tratando-se de um estudo retrospetivo de análise documental desenvolvido numa reduzida e homogénea amostra, os resultados obtidos não se podem extrapolar para o universo de fumadores que recorrem a consultas de cessação tabágica, quer nos cuidados primários quer secundários, em Portugal; contudo, podem e devem servir de ponto de partida para investigações futuras no intuito de aprofundar as questões aqui levantadas.

Além do eventual papel impulsionador para outros estudos, o presente trabalho mostrou que ainda existem algumas barreiras que dificultam a investigação nesta área; entre as mais importantes, destaca-se a inexistência de um sistema uniformizado e informatizado de registo das atividades realizadas no âmbito dos programas de apoio intensivo. De acordo com o relatório da Direção-Geral da Saúde acerca da caracterização das consultas de cessação tabágica de 2013,18 "a maior parte das consultas $(68,8 \%)$ realiza os seus registos em papel e não chegam a $22 \%$ as consultas que têm disponível em sistema eletrónico de registo".

Também nesta investigação foi possível desvendar algumas falhas do próprio programa, nomeadamente no que diz respeito à ficha de avaliação global do fumador. Esta constitui o pilar da primeira consulta dos programas de apoio intensivo à cessação e, embora esteja bem estruturada e pormenorizada em alguns parâmetros, carece de maior detalhe noutros, pelo que merece uma atualização baseada na evidência atual.

Assim sendo, e no intuito de melhorar a qualidade dos programas e reduzir o abandono precoce, as sugestões finais assentam na criação de um sistema de registo clínico informático no intuito de: simplificar e uniformizar a abordagem nas consultas, garantir a assiduidade dos utentes e facilitar o seguimento a curto e longo prazo; mas também na revisão e atualização de alguns parâmetros que constam da ficha de avaliação global do fumador, como a caracterização do suporte social, agregado familiar e estado civil. É também essencial insistir na disponibilidade de equipas multidisciplinares, principalmente para subgrupos específicos de fumadores ou, em caso de impossibilidade, garantir a fácil comunicação interpares (e.g., médico da consulta de cessação tabágica-psicólogo-psiquia- tra) para discussão dos «fumadores difíceis», no intuito de melhorar e personalizar a prestação de apoio intensivo.

Para diminuir as desistências que se registam na fase inicial é igualmente imprescindível garantir a correta motivação, capacitação e preparação de todos os fumadores e a existência de suporte social disponível para ajudar a ultrapassar os obstáculos sentidos, particularmente nos primeiros dias do processo. Da parte da equipa das consultas é essencial: transmitir uma completa disponibilidade para esclarecer todas as dúvidas e receios; disponibilizar horários flexíveis para responder às necessidades dos diferentes tipos de fumador, garantindo, por exemplo, a existência de dois horários de consulta, um no período da manhã, outro no período pós-laboral; reforçar a importância e monitorizar a assiduidade dos fumadores às consultas, particularmente às de primeira vez, de forma automática através de lembretes da marcação de consulta via SMS ou $e$-mail ou através do apoio de um administrativo para efetuar contacto telefónico no dia anterior à marcação.

\section{REFERÊNCIAS BIBLIOGRÁFICAS}

1. Direcção-Geral da Saúde. Cessação tabágica: programa tipo de actuação. Lisboa: Gradiva; 2007. ISBN 9789896162399

2. World Health Organization. Enforcing bans on tobacco advertising, promotion and sponsorship: WHO report on the global tobacco epidemic, 2013. Geneva:WHO; 2013. ISBN 9789240691605.

3. Nunes EM. Aconselhamento médico na prevenção da doença e promoção da saúde: análise a propósito do consumo de tabaco [Dissertation]. Lisboa: Escola de Saúde Pública; 2011.

4. Nunes E. Programa nacional para a prevenção e controlo do tabagismo, 2012-2016. Lisboa: Direção-Geral da Saúde; 2013. ISBN 9789726751991

5. Fiore MC, Jaén CR, Baker TB, Bailey WC, Benowitz NL, Curry, SJ et al. Treating tobacco use and dependence: 2008 update - clinical practice guideline [Internet]. Rockville, MD: U.S. Department of Health and Human Services; 2008 May. Available from: https://bphc.hrsa.gov/buckets/treatingtobacco.pdf

6. Instituto Nacional de Estatística. Inquérito nacional de saúde 2014: mais de metade da população com 18 ou mais anos tinha excesso de peso [Internet]. Lisboa: INE; 2015 Nov. Available from: https://www.ine.pt/xpor$\mathrm{tal} / \mathrm{xmain}$ ?xpid=INE\&xpgid=ine_destaques\&DESTAQUESdest_boui=2247 33757\&DESTAQUESmodo=2

7. Soares I, Carneiro AV. Smoking cessation: evidence-based recommendations. Rev Port Cardiol. 2002;21(2):203-15.

8. Aguiar M, Todo-Bom F, Felizardo M, Macedo R, Caeiro F, Sotto-Mayor R, et al. Four years' follow up at a smoking cessation clinic. Rev Port Pneumol. 2009;15(2):179-97.

9. Pacheco C, Vale A, Guimarães M, Pascoal I. Evolução do comportamento tabágico após abandono precoce de um programa de cessação tabágica [Poster]. In: $19^{\circ}$ Congresso de Pneumologia do Norte, Porto, 29 de fevereiro a 3 de março de 2012. Available from: http://hdl.handle.net/10400.23/637

10. Rebelo L. Consulta de cessação tabágica no Centro de Saúde de Alvalade: os primeiros 184 pacientes fumadores (avaliação de resultados) [Smoking cessation servisse in Alvalade health center (Lisbon): the first 184 smoking 
patients (evaluation of results)]. Rev Port Clin Geral. 2008;24(1):13-20. Portuguese

11. Dias C, Mina F. Quando as medicinas interna e familiar se unem... consulta de cessação tabágica: estudo retrospectivo de 2 anos [When general practice and Internal Medicine work as a team-smoking cessation clinic: a 2-year retrospective study]. Rev Port Med Intern. 2012;19(4):175-82. Portuguese

12. Despacho n. ${ }^{\circ} 14.202-A / 2016$, de 25 de novembro. Diário da República. $2^{a}$ Série(227).

13. Rosendo I, Fonseca G, Guedes AR, Martins V, Alegre A, Mota MA, et al. A characterisation of smokers and factors influencing motivation to stop smoking. Rev Port Pneumol. 2009;15(5):783-802.

14. Rondina RC, Gorayeb R, Botelho C. Psychological characteristics associated with tobacco smoking behavior. J Bras Pneumol. 2007;33(5):592-601. English

15. Neves A. Experiência de utentes em cessação tabágica: alguns contributos para a promoção da saúde [Dissertation]. Lisboa: Escola Nacional de Saúde Pública; 2002.

16. Park EW, Tudiver F, Schultz JK, Campbell T. Does enhancing partner support and interaction improve smoking cessation? A meta-analysis. Ann Fam Med. 2004;2(2):170-4.
17. Cohen S, Lichtenstein E. Partner behaviors that support quitting smoking. J Consult Clin Psychol. 1990;58(3):304-9.

18. Nunes E, Narigão M, editors. Relatório de caracterização das necessidades formativas dos profissionais das consultas de apoio intensivo à cessação tabágica: programa nacional para a prevenção e controlo do tabagismo (PNPCT). Lisboa: Direção-Geral da Saúde; 2013.

\section{CONFLITOS DE INTERESSE}

Os autores declaram não existir quaisquer conflitos de interesse.

\section{FONTES DE FINANCIAMENTO}

Os autores declaram que o estudo não foi financiado.

\section{ENDEREÇO PARA CORRESPONDÊNCIA}

Sofia Belo

E-mail: sofiabelo7@gmail.com

Recebido em 18-02-2017

Aceite para publicação em 12-01-2018

\section{ABSTRACT}

\section{EARLY ABANDONMENT OF TOBACCO CESSATION CONSULTATIONS AT USF DO PARQUE}

Aim: To characterize patients of smoking cessation program at USF do Parque (ACeS Lisboa Norte), describing their progress throughout the consultations with highlight to the early abandonment rate (percentage of dropouts after the first appointment) and to identify the factors associated with early abandonment.

Type of study: Retrospective observational study, based on analysis of clinical records.

Location: USF do Parque - ACeS Lisboa Norte.

Population: We studied all smokers (83) that enrolled the tobacco cessation program in 2013.

Methods: Data were obtained through document analysis of clinical records from tobacco cessation consultations. A comparative analysis was conducted between the two groups of patients according to their behavior after the first appointment - those who dropout the tobacco cessation program after the first consultation versus those who remained in the program - and statistical tests were applied (Chi-Square and Fisher's Exact Test) with a confidence interval of 95\%.

Results: Sample comprising 83 smokers, with a slight predominance of males (50.6\%) and a mean age of 47.8 years old, most of them holding a graduate degree and employed. The onset of tobacco use dates from adolescence (mean 16.2 years), with current use around 20 cigarettes per day. A moderate level of nicotine dependence was found in 59.0\% of smokers. Regarding motivation levels, the moderate level was also predominant (58.7\%). Approximately one-third dropped out the program after the first visit (early abandonment). There was a statistically significant association between early abandonment and the variables 'presence of psychopathology' $(p=0.02)$ and 'family problems' ( $p=0.03)$.

Conclusions: Intensive smoking cessation programs require continuous, regular and often prolonged contact between physician and smoker, but dropouts are common particularly after the first consultation. In the literature, both psychopathology and family problems were known to be associated with the success of the intervention; however this study showed that they also have a short-term impact on early abandonment. This result emphasises the importance of the first contact between physician and smoker and reflects the need to improve overall assessment of the smoker and to optimise strategies of support and follow-up in order to assure smokers' attendance.

Keywords: Tobacco; Consultations; General practice; Smoking cessation. 